\title{
Obtención de un material vitrocerámico a partir de una escoria de acería mezclada con vidrio de desecho
}

\author{
D. OZIEL MÉNDEZ GUERRERO, B. ALICIA VÁZQUEZ MÉNDEZ, A. ÁLVAREZ MÉNDEZ
}

Laboratorio de Materiales III. Facultad de Ciencias Químicas. Universidad Autónoma de Nuevo León. México.

\begin{abstract}
En el presente trabajo se realizó la caracterización cualitativa, cuantitativa y térmica de una escoria de acería y un vidrio de desecho, ambos de elevada producción en la zona noreste de México, para utilizarlos como materia prima en la obtención de un material vitrocerámico. Se controló el tamaño de partícula a $\leq 75$ micrómetros y se ubicaron los componentes mayoritarios de la escoria en un diagrama de equilibrio de fases, para proponer una temperatura de fusión que condujera al vidrio de partida. Posteriormente se realizaron los tratamientos térmicos para la obtención del vitrocerámico. Los materiales obtenidos se caracterizaron por difracción de rayos- $X$, método de polvos (DRX), análisis térmico diferencial-gravimétrico (ATD-TG), microscopía óptica de luz reflejada (MOLR) y microscopía electrónica de barrido (MEB). Finalmente, se realizaron ensayos de microdureza Vickers y de resistencia al ataque químico que permitieron proponer una aplicación para el vitrocerámico.
\end{abstract}

\section{Palabras clave: Escoria, casco de vidrio, vitrocerámico.}

\section{Obtaining a glass-ceramic material from a steel slag mixed with glass cullet}

In this paper, the qualitative, quantitative and thermal characterization of a steel slag and glass cullet of high generation rate in northern Mexico were made in order to use these wastes as raw materials in the production of glassceramics. The particle size was controlled at sizes $\leq 75$ micrometers and the major components of the slag were located in a phase equilibrium diagram for proposing a reaction temperature that leaded to the starting glass. Later, heat treatments were performed to obtain the glassceramics. The materials were characterized by powder X-ray diffraction (XRD), differential thermal analysis coupled with thermal gravimetric analysis (DTA-TGA), reflected light optical microscopy (RLOM) and scanning electron microscopy (SEM). Subsequently, Vickers microhardness and chemical resistance tests were performed, which enabled us to propose an application of the glassceramics.

Keywords: Slag, cullet, glassceramics.

\section{INTRODUCCIÓN}

La ciencia y la tecnología de los materiales han cobrado un gran auge en la última década debido a las demandas que han surgido en la sociedad por resolver problemas presentados a nivel mundial y regional de tipo energético, reutilización o reciclaje de residuos industriales y de carácter ambiental, por mencionar algunos. La mayor eficiencia y competitividad económica que los sectores productivos y de protección al medio ambiente se exigen cada día, también ha contribuido a este gran desarrollo ${ }^{(1)}$.

Las industrias siderúrgicas y metalúrgicas han acelerado su producción gracias a estos avances tecnológicos y científicos, lo que ha aumentado la producción de su residuo mayoritario, las escorias (se generan $0,55 \mathrm{~kg}$ de escoria por cada $\mathrm{kg}$ de acero producido), cuya composición química y física depende del mineral usado y de la tecnología empleada (2).

Por otro lado, la industria del vidrio también es una gran generadora de residuos, ya sea por autoconsumo por el propio proceso de fabricación o debido a la insuficiente calidad de los productos. En esta industria el vidrio de desecho es llamado "casco" y se estima que la producción de casco de vidrio en las industrias vidrieras es del 16,93\% del total de vidrio bruto fundido, a esto hay que agregarle todos aquellos productos de vidrio que se desechan por la comunidad (deshecho de otras industrias, doméstico, centros de educación, construcción, etc.) (3).

Debido al descuido del ser humano en nuestro planeta se han presentado varios problemas, la contaminación y la escasez de ciertos elementos como el agua dulce, petróleo y carbón se pueden considerar como los más importantes. Todos estos problemas que se han acarreado por la mano del ser humano es el motivo de que la industria del reciclaje haya crecido a pasos agigantados. El reciclaje lo podemos describir como una operación compleja que permite la recuperación, transformación y elaboración de un nuevo material a partir de residuos, ya sea total o parcialmente de acuerdo a la composición definitiva del nuevo material. La transformación de desechos en nuevos productos tiene múltiples beneficios: recuperación de los terrenos donde se almacenan y disminución del costo de almacenaje, minimiza la explotación de recursos vírgenes no renovables, reduce la posibilidad de generar problemas de salud en las zonas 
aledañas, ingreso de divisas por medio de la generación del nuevo producto, entre otros ${ }^{(2 \mathrm{y} 4)}$.

La industria del reciclaje es muy extensa, y comprende desde la recolección de la materia prima hasta la entrega del producto total, listo para ser comercializado, independientemente del material a reciclar.

Es aquí donde el proceso vítreo y vitrocerámico perfilan como nuevas oportunidades para el reciclaje de materiales de desecho; sin embargo, el máximo reto consiste en demostrar la utilidad del proceso y producir materiales que posean características fisicoquímicas capaces de competir con las existentes en el mercado, y de esta forma impactar positivamente el medio ambiente con la desaparición de los depósitos de tales residuos y con el ahorro de recursos y materias primas naturales ${ }^{(5-15)}$.

\section{MATERIALES Y MÉTODOS}

Las materias primas del presente estudio fueron escoria de acería proveniente del alto horno de una industria de la localidad (Monterrey, Nuevo León), proporcionada por la misma industria, y casco de vidrio obtenido de botellas de jugo, provenientes de desecho doméstico. Dichos materiales se sometieron a un control de tamaño de partícula $\leq 75 \mu \mathrm{m}$ en un molino de tungsteno y un tamiz No. 200.

El análisis mineralógico de los materiales se realizó utilizando un difractómetro de rayos-X Siemens D-5000 con radiación monocromática $\mathrm{Cu}_{\mathrm{k} \alpha}(\lambda=1,5418 \AA)$, filtro de $\mathrm{Ni}$, de $0^{\circ}$ a $90^{\circ}$ en $2 \theta$ con un paso de $0,01^{\circ}$ y un tiempo de $0,3 \mathrm{~s}$, ajustando la energía del tubo en $35 \mathrm{kV}$ y $25 \mathrm{~mA}$.

Los análisis térmicos se llevaron a cabo en un analizador térmico (ATD-ATG) TAInstruments SDT 2960 que incorpora un controlador de temperatura Netzsch TASC 414/ 2 controlado por un programa computarizado, empleando como material de referencia $\alpha-\mathrm{Al}_{2} \mathrm{O}_{3}$ calcinada adecuadamente.

La temperatura máxima de trabajo fue de $1300{ }^{\circ} \mathrm{C}$ y la velocidad de calentamiento empleada fue de $10{ }^{\circ} \mathrm{C} / \mathrm{min}$ con un flujo de aire de $100 \mathrm{~mL} / \mathrm{min}$.

El análisis químico cuantitativo de la escoria se realizó mediante un espectrómetro de absorción atómica marca GBC Scientific modelo 932 AA, que utiliza una mezcla de aireacetileno para la llama del quemador y lámparas de cátodo

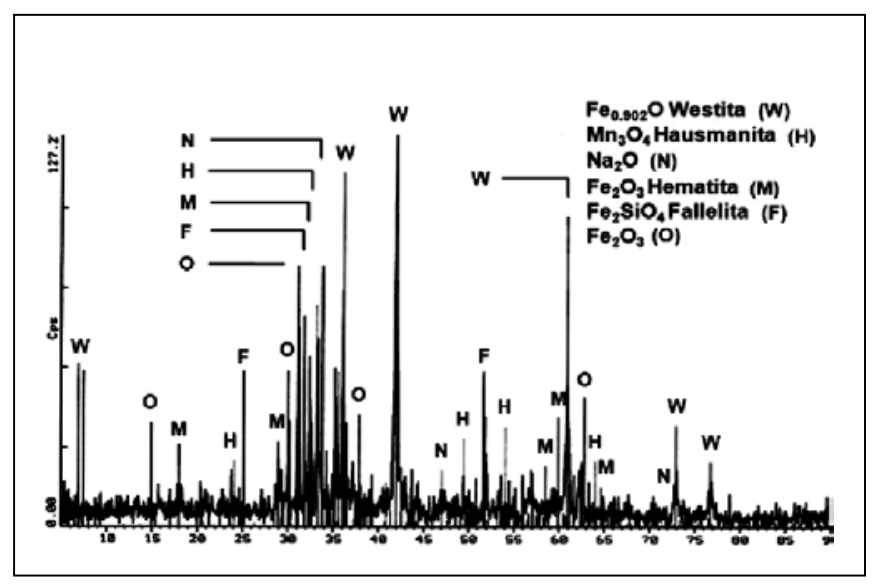

Figura 1. Caracterización mineralógica de la escoria de acería por difracción de rayos- $X$ (método de polvos). hueco para la determinación de los analitos. El contenido de carbono se determinó mediante un analizador elemental Perkin Elmer 2400, equipado con una columna cromatográfica a $975{ }^{\circ} \mathrm{C}$ con alimentación de oxígeno puro y utilizando helio (He) como gas portador de los productos de combustión. Para determinar el contenido de $\mathrm{SiO}_{2}$ se utilizó un método gravimétrico de calcinación a $1200{ }^{\circ} \mathrm{C}$ por 6 horas.

El análisis cualitativo y cuantitativo del casco de vidrio se realizó por fluorescencia de rayos-X (FRX) empleando un espectrómetro Phillips modelo PW-2424 con tubo de rayos-X de ventana ultrafina y ánodo de rodio $(\mathrm{Rh})$ de alta potencia a $2,4 \mathrm{~kW}$.

Los vidrios de partida se obtuvieron por fusión, en crisol de platino $(\mathrm{Pt})$, de mezclas escoria-casco de vidrio a 1450 ${ }^{\circ} \mathrm{C}$ por 2 horas en horno eléctrico y vaciado en placa fría de acero inoxidable. Los vitrocerámicos se obtuvieron por tratamiento térmico de los vidrios de partida a la temperatura de cristalización marcada por el ATD, usando una rampa de calentamiento de $10{ }^{\circ} \mathrm{C} / \mathrm{min}$ y estableciendo un tiempo de residencia de 2 horas a dicha temperatura, posterior a lo cual, se realizó un enfriamiento rápido de las muestras.

Una vez obtenidos los vitrocerámicos, fueron caracterizados al igual que el vidrio de partida, por microscopía electrónica de barrido con sistema de dispersión de energías (MEB-EDS) usando un equipo Jeol 6490 LV. Para realizar este análisis se embutieron tanto los vitrocerámicos como el vidrio de partida en una resina acrílica de secado en frío, para después pulirlas usando lijas No. 180, 320, 500 y 1400 y finalmente lavarlas en etanol con ultrasonido. Posteriormente las muestras fueron metalizadas utilizando una tinta de plata $(\mathrm{Ag})$ con el fin de volverlas conductoras.

Para los ensayos de dureza se empleó un microdurómetro Shimadzu 4498 con enfoque óptico y temporizador integrado, realizando 15 indentaciones por muestra y aplicando una carga $(\mathrm{P})$ de $200 \mathrm{Kg}_{\mathrm{f}}$ por $15 \mathrm{~s}$. Las huellas de indentación obtenidas $(d)$ se promediaron para calcular la dureza Vickers del material utilizando la fórmula:

$$
\mathrm{HV}=1420\left(\mathrm{P} / d^{2}\right)
$$

La determinación de la resistencia química de los materiales se realizó ante soluciones de $\mathrm{HCl}$ y $\mathrm{NaOH}$ al $5 \%$ por un periodo de 24 horas y evaluando la pérdida de masa.

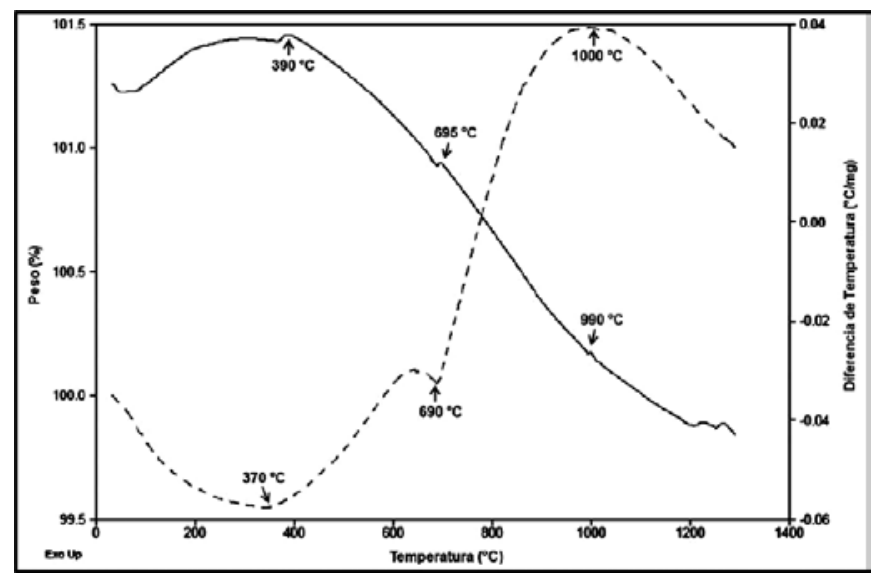

Figura 2. Análisis térmico diferencial y termogravimétrico de la escoria de acería. (-ATD --ATG) 


\section{RESULTADOS Y DISCUSIÓN}

El análisis mineralógico de la escoria se muestra en el difractograma de la Figura 1, donde se observa un elevado grado de cristalinidad comparada con otras escorias, que por lo regular son amorfas, esta escoria tiene la presencia de óxidos de hierro, manganeso y sodio, además de un silicato de hierro.

El análisis térmico de la escoria se muestra en la Figura 2, donde se observa claramente un descenso en la masa de la muestra al inicio del análisis, esta pérdida de masa se atribuye a la humedad presente en la escoria. A $390{ }^{\circ} \mathrm{C}$ y $695{ }^{\circ} \mathrm{C}$ se observan dos picos exotérmicos asociados a incrementos en la curva del cambio de la masa de la muestra a $370{ }^{\circ} \mathrm{C}$ y 690 ${ }^{\circ} \mathrm{C}$, dichos eventos los podemos atribuir a la oxidación de compuestos presentes en la escoria como el manganeso y el hierro, el pico exotérmico de $990{ }^{\circ} \mathrm{C}$ posiblemente evidencie un cambio de fase en alguno de minerales presentes, y la pérdida de masa que se presenta a $1000{ }^{\circ} \mathrm{C}$, se atribuye al desprendimiento del carbón o azufre que posiblemente se encuentre ocluido dentro de la estructura de la escoria y que es liberado por efecto de la temperatura.

El análisis químico realizado a la escoria se muestra en la Tabla I, donde se observan los elementos (en forma de óxido) presentes, siendo los mayoritarios $\mathrm{Fe}, \mathrm{Mn}, \mathrm{Na}, \mathrm{Si}$ y $\mathrm{Al}$; sin embargo, solo el $\mathrm{Fe}, \mathrm{Mn}$, $\mathrm{Na}$ y $\mathrm{Si}$ coinciden con fases encontradas en el difractograma de rayos-X.

TABLA I. CARACTERIZACIÓN CUANTITATIVA COMPLETA DE LA ESCORIA.

\begin{tabular}{|c|c|c|c|}
\hline Elemento & $\%$ Óxido & Elemento & \% Óxido \\
\hline $\mathrm{Fe}$ & $44,60 \%$ & $\mathrm{Ca}$ & $2,64 \%$ \\
\hline $\mathrm{Cd}$ & $0,04 \%$ & $\mathrm{~Pb}$ & $0,05 \%$ \\
\hline $\mathrm{Zn}$ & $0,03 \%$ & $\mathrm{Cr}$ & $0,34 \%$ \\
\hline $\mathrm{Cu}$ & $0,04 \%$ & $\mathrm{Mg}$ & $3,15 \%$ \\
\hline $\mathrm{Mn}$ & $16,40 \%$ & $\mathrm{Na}$ & $18,53 \%$ \\
\hline $\mathrm{K}$ & $0,05 \%$ & $\mathrm{Ni}$ & $0,09 \%$ \\
\hline $\mathrm{Al}$ & $6,25 \%$ & $\mathrm{C}$ & $0,26 \%$ \\
\hline $\mathrm{Si}$ & $7,53 \%$ & & \\
\hline $\mathrm{TOTAL}$ & & $100,00 \%$ & \\
\hline
\end{tabular}

A partir de su composición química, es posible ubicar la escoria en el diagrama ternario de equilibrio de fases $\mathrm{FeO}-$ $\mathrm{Na}_{2} \mathrm{O}-\mathrm{SiO}_{2}$ para determinar la factibilidad de formación del vidrio y el tratamiento térmico requerido (Figura 3). Para ello, se recalculó al $100 \%$ los porcentajes en peso de los mayoritarios en la escoria (Tabla II).

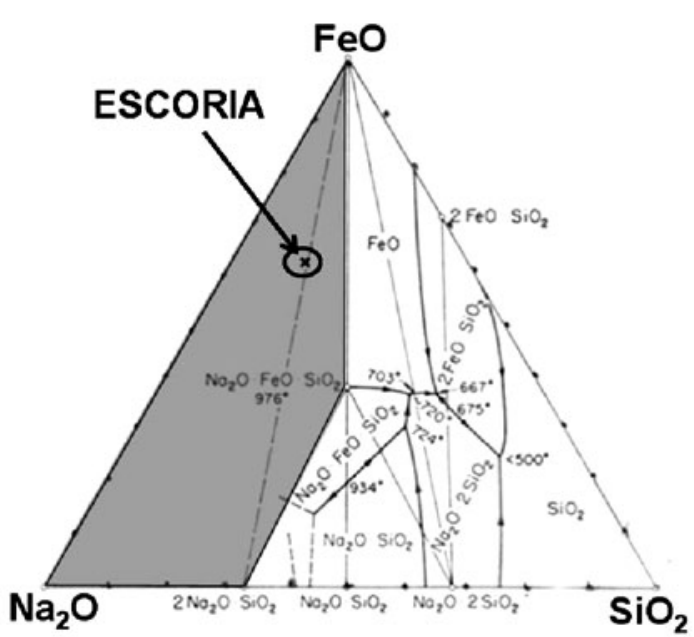

Figura 3. Ubicación de la escoria en el diagrama de fases $\mathrm{FeO}_{-} \mathrm{SiO}_{2}-$ $\mathrm{Na}_{2} \mathrm{O}$.

Tabla II. Datos obtenidos al ReCALCUlaR los Óxidos FeO, $\mathrm{NA}_{2} \mathrm{O}$ Y $\mathrm{SiO}_{2}$ PARA SU UBICACIÓN EN EL DIAGRAMA DE FASES.

\begin{tabular}{|c|c|c|}
\hline Óxido de metal & $\%$ & $\begin{array}{c}\text { Recalculación } \\
\text { al 100\% }\end{array}$ \\
\hline $\mathrm{FeO}$ & 44,60 & 63,12 \\
\hline $\mathrm{Na}_{2} \mathrm{O}$ & 18,53 & 26,22 \\
\hline $\mathrm{SiO}_{2}$ & 7,53 & 10,66 \\
\hline$\underline{\text { Total }}$ & $\mathbf{7 0 , 6 6}$ & $\mathbf{1 0 0 , 0 0}$ \\
\hline
\end{tabular}

En el diagrama se observa que la escoria se encuentra ubicada en la línea de compatibilidad del intermediario $2 \mathrm{Na}_{2} \mathrm{O} \bullet \mathrm{SiO}_{2}$ con el $\mathrm{FeO}$, por lo que se pensó factible la formación de la red vítrea a una temperatura adecuada.

La temperatura seleccionada para la fusión de la escoria fue $1450{ }^{\circ} \mathrm{C}$ con 2 horas de residencia, por ser las condiciones convencionales para la obtención de vidrios, utilizando una rampa de calentamiento de $10{ }^{\circ} \mathrm{C} / \mathrm{min}$; sin embargo, no se logró obtener el material en estado vítreo, por lo que se

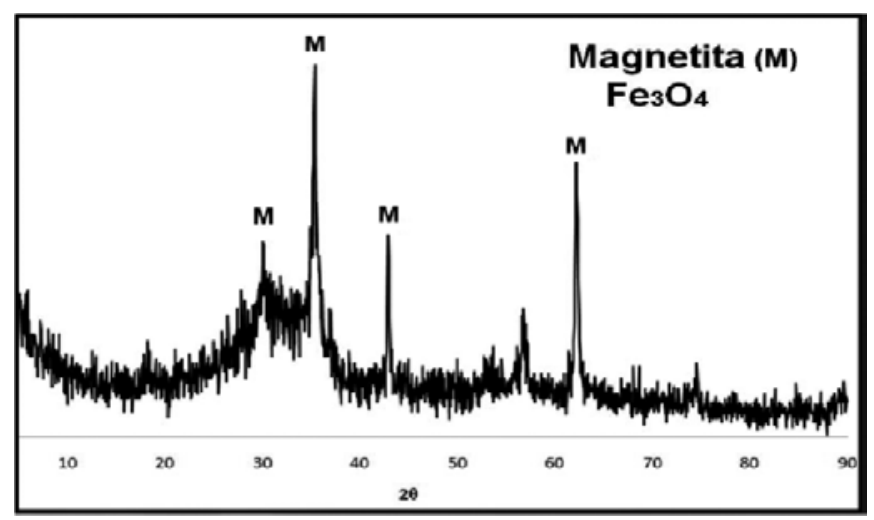

Figura 4. Difractograma de la escoria tratada a $1650^{\circ} \mathrm{C}$ por dos horas. 
hicieron otros dos experimentos a 1550 y $1650^{\circ} \mathrm{C}$, de los cuales no se obtuvo un resultado satisfactorio.

En la Figura 4 se muestra el difractograma de rayos- $X$ del material obtenido por fusión a $1650^{\circ} \mathrm{C}$, donde se observa que el material no es completamente amorfo, ya que aparecen picos representativos de la magnetita $\left(\mathrm{Fe}_{3} \mathrm{O}_{4}\right)$.

Debido a que trabajar a estas temperaturas no es costeable económica ni energéticamente, se procedió a la adición del casco de vidrio, para lo cual se realizó un análisis por fluorescencia de rayos-X para determinar su composición cualitativa y cuantitativa; los resultados se muestran en la Tabla III

TABLA III. CARACTERIZACiÓN CUALITATIVA Y CUANTITATIVA DEL CASCO DE VIDRIO.

\begin{tabular}{|c|c|c|c|}
\hline Óxido & $\%$ masa & Óxido & $\%$ masa \\
\hline $\mathrm{SiO}_{2}$ & 71,15 & $\mathrm{SO}_{3}$ & 0,21 \\
\hline $\mathrm{CaO}$ & 14,09 & $\mathrm{TiO}_{2}$ & 0,15 \\
\hline $\mathrm{Na}_{2} \mathrm{O}$ & 12,23 & $\mathrm{Fe}_{2} \mathrm{O}_{3}$ & 0,12 \\
\hline $\mathrm{Al}_{2} \mathrm{O}_{3}$ & 0,98 & $\mathrm{MgO}$ & 0,09 \\
\hline $\mathrm{ZrO}_{2}$ & 0,02 & $p p c$ & 0,64 \\
\hline $\mathrm{K}_{2} \mathrm{O}$ & 0,32 & Total & 100,00 \\
\hline
\end{tabular}

${ }^{*} \mathrm{ppc}=$ pérdidas por calcinación

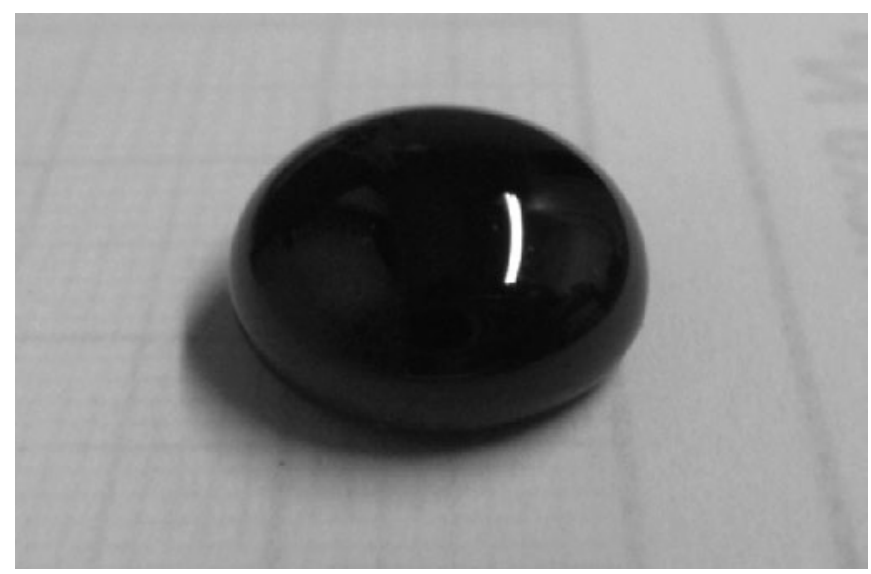

Figura 4. Fotografía del vidrio obtenido de la mezcla al 50\% de escoria y casco de vidrio.

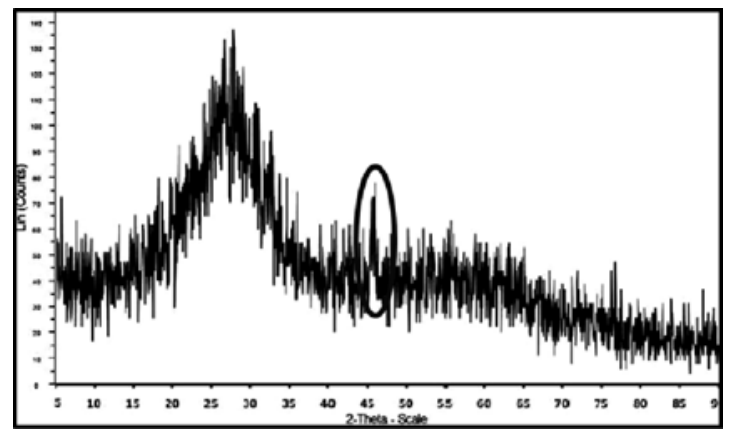

Figura 5. Difractograma de rayos- $X$ en polvo de la mezcla al 50\% de escoria y casco de vidrio.
Una vez caracterizado el casco de vidrio, se calculó el porcentaje de escoria y casco necesario para la obtención de un vidrio con $70 \%$ de óxidos formadores de red $\left(\mathrm{SiO}_{2}\right.$ y $\left.\mathrm{Fe}_{2} \mathrm{O}_{3}\right)$ y $30 \%$ de modificadores de red $\left(\mathrm{CaO}\right.$ y $\left.\mathrm{Na}_{2} \mathrm{O}\right)$, composición convencional de los vidrios. ${ }^{(16-18)}$

Los resultados revelaron necesaria una composición de $58,63 \%$ de escoria y $41,37 \%$ de casco de vidrio. En base al resultado anterior se propuso una mezcla al $50 \%$, la cual fue sometida a un tratamiento térmico a $1450{ }^{\circ} \mathrm{C}$ por 2 horas con una rampa de calentamiento de $10{ }^{\circ} \mathrm{C} / \mathrm{min}$ para lograr su fusión y vaciar en placa. Se obtuvo un vidrio negro, muy brillante y con apariencia de espejo (Figura 4); que presentaba una mancha color café, evidencia de cristalización superficial e inestabilidad en el material. El difractograma obtenido de este vidrio se muestra en la Figura 5, donde se observa un espectro amorfo, pero destaca un pequeño pico que corrobora la cristalización supuesta.

Se realizó una nueva mezcla, ahora utilizando $45 \%$ escoria y $55 \%$ casco de vidrio, bajo las mismas condiciones de reacción que la mezcla al $50 \%$ obteniendo un material negro, muy brillante y de apariencia de espejo, pero sin presentar cristalización superficial (Figura 6); esto se corrobora analizando el difractograma de rayos $\mathrm{X}$ de dicho material, donde se observa un espectro completamente amorfo (Figura 7).

Éste ultimo vidrio se eligió como precursor para la obtención de materiales vitrocerámicos, debido a la mayor

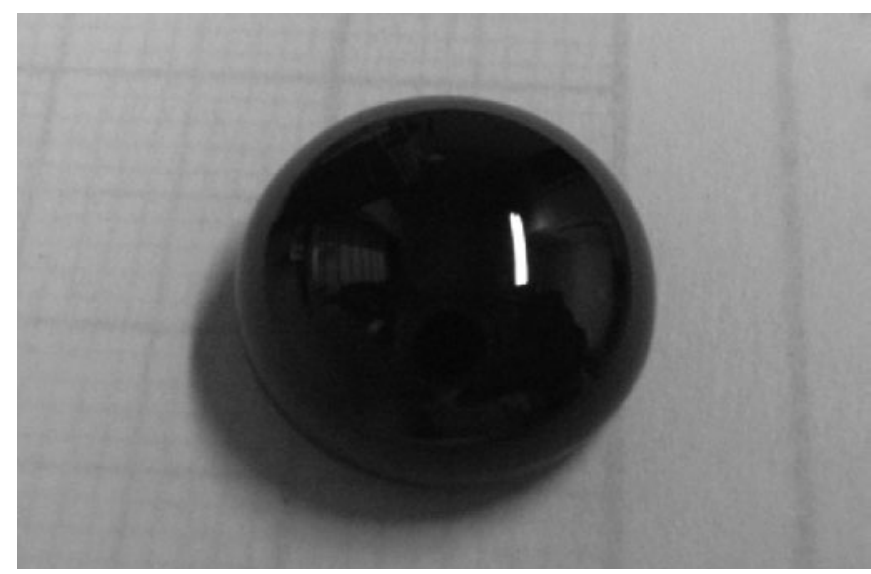

Figura 6. Fotografía del vidrio obtenido a partir de una mezcla escoriacasco de vidrio $45-55 \%$ en peso.

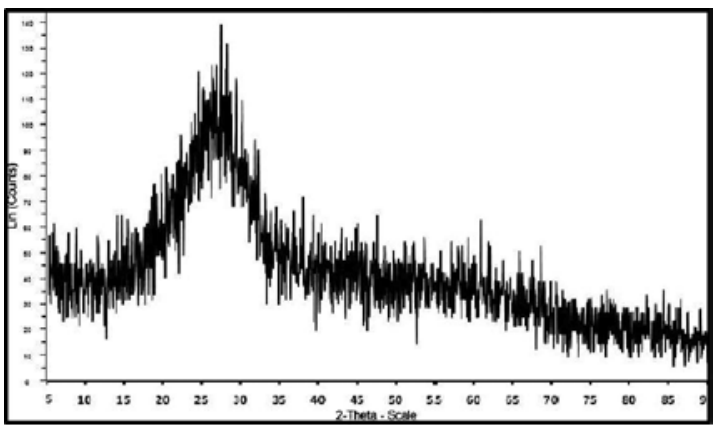

Figura 7. Difractograma de rayos-X en polvo del vidrio obtenido en la composición escoria-casco 45-55\% en masa. 
estabilidad, de acuerdo al difractograma de rayos- $X$ que no presentó indicios de cristalización.

Por lo anterior se realizó un análisis térmico diferencial y gravimétrico (Figura 8), para caracterizar térmicamente el vidrio y obtener la(s) temperatura(s) de cristalización del material.

En la curva de ATD podemos observar la temperatura de transformación vítrea $\left(\mathrm{T}_{\mathrm{g}}\right)$ a $610{ }^{\circ} \mathrm{C}$ y dos picos exotérmicos a 806 y $840{ }^{\circ} \mathrm{C}$, correspondientes a picos de cristalización. Se realizaron tratamientos térmicos a estas dos temperaturas con un tiempo de residencia de 2 horas, utilizando una rampa de calentamiento de $10^{\circ} \mathrm{C} / \mathrm{min}$ y una vez transcurrido el tiempo de residencia se enfriaron rápidamente las muestras para "congelar" las fases desarrolladas.

Para el tratamiento a $806{ }^{\circ} \mathrm{C}$ se obtuvo un material con textura rugosa, de color variado entre verde y café, y con brillo tipo tornasol (Figura 9). A dicho material se le realizó un análisis por difracción de rayos- $X$ en método de polvos para analizar su mineralogía (Figura 10). En el análisis obtenido se observa un espectro amorfo que representa una cantidad de fase vítrea considerable y a cuya permanencia atribuimos el brillo peculiar del material obtenido; el análisis también reveló las fases cristalizadas en el vidrio, producto del tratamiento térmico aplicado, la parte cristalizada se compone principalmente de jacobskita, magnetita y aegirina.

El tratamiento a $840{ }^{\circ} \mathrm{C}$ concluyó en un material opaco, color café (Figura 11), sin la textura rugosa ni brillo del

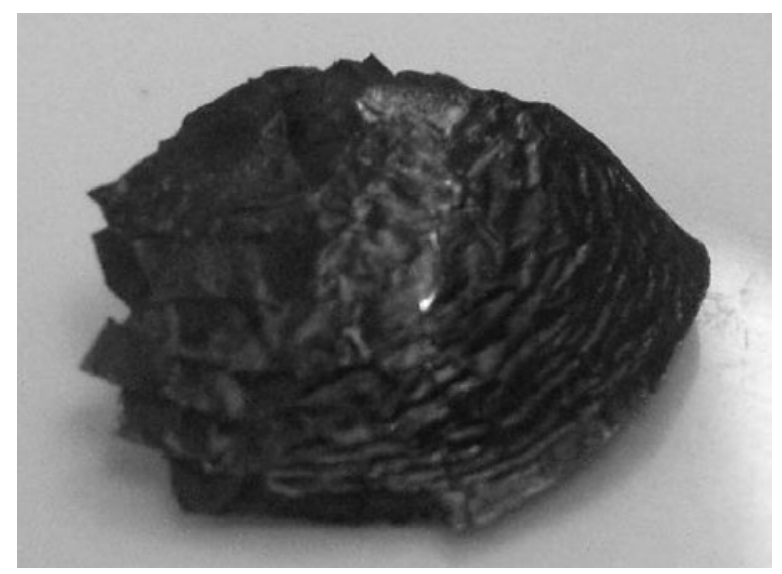

Figura 9. Imagen del material obtenido con el tratamiento térmico de cristalización de $806^{\circ} \mathrm{C}$.

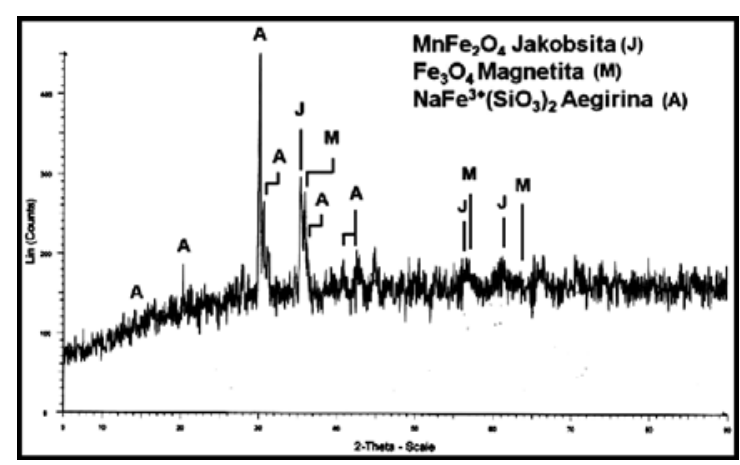

Figura 10. Difractograma de rayos-X en polvo para el vidrio tratado a $806^{\circ} \mathrm{C}$.

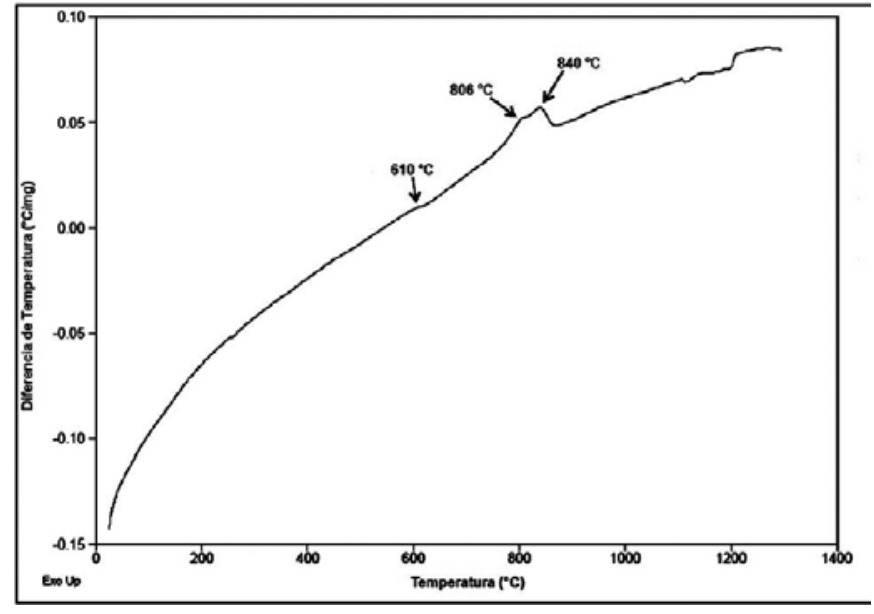

Figura 8. Análisis térmico diferencial del vidrio obtenido a partir de la composición escoria-casco $45-55 \%$ en peso.

material obtenido a $840^{\circ} \mathrm{C}$. Este nuevo material se analizó por difracción de rayos- $X$ en método de polvos (Figura 12), donde se identificaron las fases wollastonita, jakobsita, magnetita, hedenbergita, aegirina y $\mathrm{AlFeO}_{3}$; además de desaparecer casi por completo el espectro amorfo observado en el material obtenido a $806^{\circ} \mathrm{C}$.

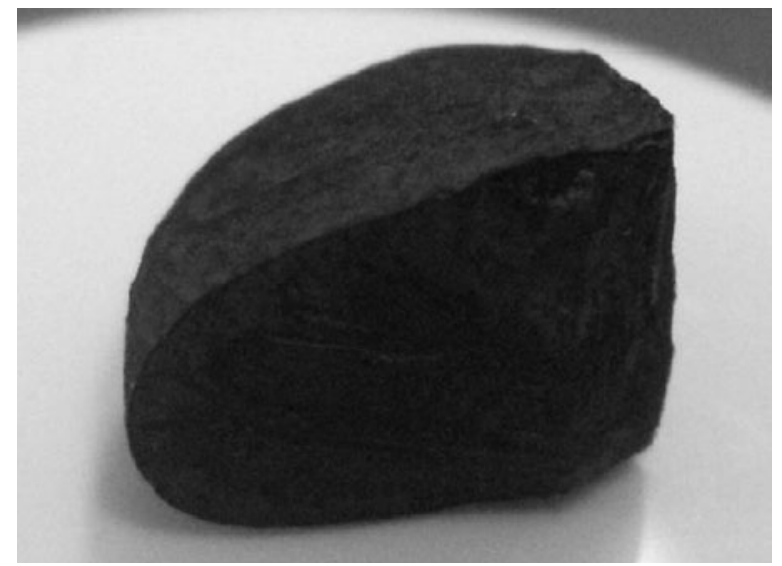

Figura 11. Imagen del material obtenido mediante el tratamiento térmico a $840{ }^{\circ} \mathrm{C}$ por 2 horas.

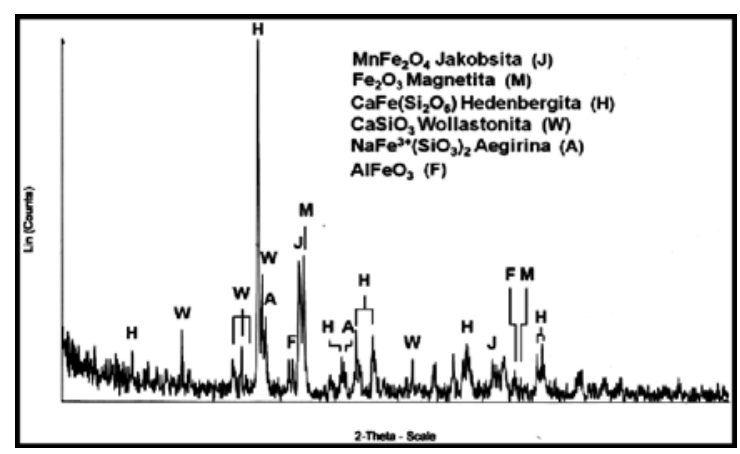

Figura 12. Difractograma de rayos-X en polvo del material obtenido a $840^{\circ} \mathrm{C}$ por 2 horas. 
Es evidente en los difractogramas la presencia de fases pertenecientes a la familia de los inosilicatos: aegerina, wollastonita y hedenbergita, ésta familia de compuestos se caracteriza por presentar propiedades mecánicas muy atractivas, por ejemplo elevada dureza, lo que hace presumible que los vitrocerámicos obtenidos tendrán una dureza mayor que la del vidrio de partida.

Las muestras se analizaron por MEB-EDS para corroborar la obtención de vitrocerámicos y compararlos con el vidrio de partida.

El vitrocerámico obtenido a $806{ }^{\circ} \mathrm{C}$ mostró una zona clara y una zona oscura en la microscopía electrónica de barrido a 3300x, Figura 13 (a), y al realizar el EDS en dichas zonas se encontró, en la zona oscura la misma composición que la encontrada en el análisis del vidrio, por lo que se supone sea matriz vítrea residual, Figura 13 (c). En las zonas claras el EDS reveló como componentes mayoritarios al silicio, oxígeno, calcio y hierro, en una proporción que corresponde a la aegirina $\mathrm{NaFe}\left(\mathrm{SiO}_{3}\right)_{2}$, Figura 13 (b). b)

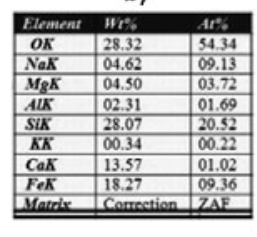

a)

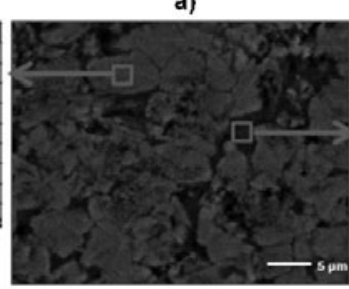

c)

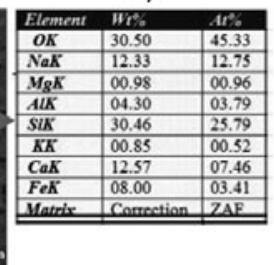

Figura 13. Microscopía electrónica de barrido a 3300x y análisis por dispersión de energías de las fases encontradas en el vitrocerámico obtenido a $806^{\circ} \mathrm{C}$. a) Micrografía general, b) fases cristalinas c) fase vítrea

Para analizar las fases cristalinas desarrolladas en el vitrocerámico tratado a $840{ }^{\circ} \mathrm{C}$ se realizó un análisis MEB a 3300x, obteniendo una micrografía general en una de las áreas del material, que se puede apreciar en la Figura 14 (a), donde se observa la formación cristalina con granos de tamaños de partícula menores a $5 \mu \mathrm{m}$ de formas irregulares. Se realizó un análisis por dispersión de energía (EDS) en diferentes zonas: en la Figura 14 (b) se encontraron relaciones estequiométricas $\mathrm{Si}: \mathrm{O}$ y Ca:Fe que sugieren se trate de la fase hedenbergita; en la Figura 14 (c) se presentan relaciones de $\mathrm{Mn}: \mathrm{Fe}: \mathrm{O}$ que se atribuyen a la presencia de la fase jakobsita, se observa también en esta zona un contenido mayor de Fe que en b) por lo que se asocia la fase magnetita, de la misma manera la cantidad de $\mathrm{Na}$, Fe y Si presentes en el análisis EDS sugiere la presencia de la fase aegirina. No fue posible determinar por separado las fases minoritarias, aunque el análisis puntual de ambas zonas sugiere que puede haber presencia de éstas, ya que podrían estar inmersas en la matriz vítrea residual presente en el vitrocerámico.

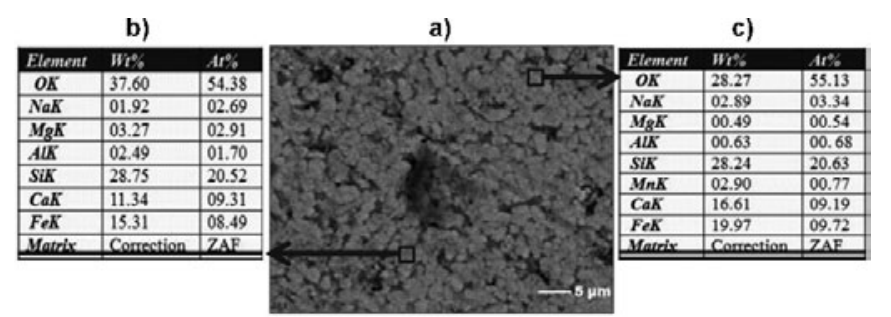

Figura 14. Micrografía electrónica de barrido 3300x del vitrocerámico $840^{\circ} \mathrm{C}$. a) Micrografía general, b) y c) análisis por dispersión de energías de fases cristalinas.

El ensayo de dureza de los materiales sinterizados proporcionó los valores de la longitud de las diagonales obtenidas para las 15 indentaciones, los cuales se promediaron para obtener, con ayuda de la fórmula anteriormente mencionada, la dureza tanto del vidrio precursor, como de los vitrocerámicos. En la Tabla IV, se resume el análisis de dureza realizado a los materiales.

Como se puede observar en dicha Tabla IV, los valores de dureza presentados por los materiales vitrocerámicos son mayores que los del vidrio de partida, además se observa que el grado de cristalinidad del material es un factor que influye en la dureza del mismo, ya que el vitrocerámico de $806^{\circ} \mathrm{C}$, que presentaba una cantidad considerable de fase vítrea residual, presentó menor dureza que el vitrocerámico de $840{ }^{\circ} \mathrm{C}$, que resultó casi completamente cristalino.

Los valores de dureza de los materiales obtenidos son elevados, tomando en cuenta la carga aplicada, $200 \mathrm{~kg}_{\mathrm{f}}$, siendo el vitrocerámico obtenido a $840{ }^{\circ} \mathrm{C}$ el que presentó los mejores valores de dureza $(1073 \mathrm{HV})$.

TABLA IV. VALORES PROMEDIO DE LAS HUELLAS DE INDENTACIÓN Y DUREZA VICKERS CALCULADA PARA LOS MATERIALES.

\begin{tabular}{|c|c|}
\hline \multicolumn{2}{|c|}{ Vidrio } \\
\hline D1 $(\mu \mathrm{m})$ & $20,17 \pm 1,82$ \\
\hline D2 $(\mu \mathrm{m})$ & $94,81 \pm 2,01$ \\
\hline Dureza (HV) & $20,02 \pm 2,06$ \\
\hline \multicolumn{2}{|c|}{ Vitrocerámico $806^{\circ} \mathrm{C}$} \\
\hline D1 $(\mu \mathrm{m})$ & $19,46 \pm 2,09$ \\
\hline D2 $(\mu \mathrm{m})$ & $966,40 \pm 146,62$ \\
\hline Dureza (HV) & $18,57 \pm 0,96$ \\
\hline \multicolumn{2}{|c|}{ Vitrocerámico $840{ }^{\circ} \mathrm{C}$} \\
\hline D1 $(\mu \mathrm{m})$ & $18,70 \pm 0,77$ \\
\hline D2 $(\mu \mathrm{m})$ & $1073,33 \pm 95,85$ \\
\hline Dureza (HV) & \\
\hline
\end{tabular}


Finalmente, los resultados del análisis por resistencia al ataque químico de los materiales estudiados ante soluciones de $\mathrm{HCl}$ y $\mathrm{NaOH}$ al $5 \%$ durante 24 horas a $25^{\circ} \mathrm{C}$, se presentan en la Tabla V.

Los resultados obtenidos demuestran que el vidrio de partida presenta gran atacabilidad a soluciones ácidas al perder más de la mitad de su masa debido al ataque ácido, sin embargo los vitrocerámicos mejoran mucho su resistencia en comparación al vidrio al reportar pérdidas de menos del $10 \%$; en el caso de la solución alcalina no se presenta mucha pérdida, todos los materiales estudiados pierden menos del $1 \%$ de su masa inicial, aún así los vitrocerámicos presentan mejor resistencia al álcali.

TABla XII. Resultados De LA PÉRDida DE PESO DE LOS MATERIALES POR ATAQUE QUÍMICO ANTE SOLUCIONES DE HCL Y NAOH AL 5\%.

\begin{tabular}{|c|c|c|c|}
\hline \multicolumn{4}{|c|}{ Vidrio } \\
\hline \multicolumn{2}{|c|}{$\mathrm{HCl}$} & \multicolumn{2}{|c|}{$\mathrm{NaOH}$} \\
\hline$m_{0}$ & 0,2380 & $m_{0}$ & 0,2930 \\
\hline$m_{f}$ & 0,1102 & $m_{f}$ & 0,2918 \\
\hline pérdida & $53,69 \%$ & pérdida & $0,41 \%$ \\
\hline \multicolumn{4}{|c|}{ Vitrocerámico $806^{\circ} \mathrm{C}$} \\
\hline \multicolumn{2}{|c|}{$\mathrm{HCl}$} & \multicolumn{2}{|c|}{$\mathrm{NaOH}$} \\
\hline$m_{0}$ & 0,3307 & $m_{0}$ & 0,3375 \\
\hline$m_{f}$ & 0,3075 & $m_{f}$ & 0,3364 \\
\hline pérdida & $7,01 \%$ & pérdida & $0,33 \%$ \\
\hline \multicolumn{4}{|c|}{ Vitrocerámico $840^{\circ} \mathrm{C}$} \\
\hline \multicolumn{2}{|c|}{$\mathrm{HCl}$} & \multicolumn{2}{|c|}{$\mathrm{NaOH}$} \\
\hline$m_{0}$ & 0,3859 & $m_{0}$ & 0,3749 \\
\hline$m_{f}$ & 0,3720 & $m_{f}$ & 0,3739 \\
\hline pérdida & $3,62 \%$ & pérdida & $0,27 \%$ \\
\hline
\end{tabular}

\section{CONCLUSIONES}

No fue posible obtener el vidrio directamente de la escoria en tres intentos a diferentes temperaturas $\left(1450,1550\right.$ y $\left.1650{ }^{\circ} \mathrm{C}\right)$.

Fue necesaria la adición de casco de vidrio a la escoria para poder disminuir la temperatura de fusión, logrando obtener el vidrio a $1450{ }^{\circ} \mathrm{C}$ con dos horas de reacción. La composición que presentó mejores resultados para la formación del vidrio fue de $45 \%$ escoria y 55\% casco de vidrio, obteniendo un vidrio oscuro (negro), muy brillante y de apariencia espejo.

El vidrio presentó dos temperaturas de cristalización al ser analizado por ATD, obteniendo al realizar tratamientos térmicos a dichas temperaturas dos materiales con características macroscópicas diferentes.
Al realizar los estudios de comparación por DRX, se encontró que las principales fases cristalinas desarrolladas fueron silicatos, especialmente de los que pertenecen a la familia de los inosilicatos, lo que pudiera atribuirle buenas propiedades mecánicas; otras fases encontradas fueron óxidos de hierro en diferentes estructuras.

Los análisis por MEB confirmaron la obtención de fases cristalinas en los vidrios tratados a las temperaturas de cristalización, y el análisis por dispersión de energías corroboró los resultados obtenidos por DRX, al identificar dos fases presentes en los difractogramas.

La evaluación de la dureza y la resistencia al ataque químico mostró mejores resultados para los vitrocerámicos respecto a los vidrios precursores, aumentando la dureza hasta en 100 unidades Vickers y reduciendo la pérdida de masa ante ataque ácido de 50 a $10 \%$.

El vitrocerámico con mejores propiedades resultó ser el obtenido a $840{ }^{\circ} \mathrm{C}$, el cual se propone como material para recubrimiento de mesas de trabajo rudo, de acuerdo a las características químicas y mecánicas presentadas.

\section{REFERENCIAS}

1. M. Martin, y J. Veciana, "Diseño y preparación de nuevos sistemas moleculares", Revista El futuro de la Química en Europa, 31. (1998)

2. X. Castells, "Reciclaje de residuos industriales: Aplicación a la fabricación de materiales para la construcción", Ediciones de los Santos, 609 pp. (2000)

3. Handbook of Glass Technology, TNO International Course on Glass Technology, Capítulo 7 Glass recycling. (2002)

4. K. Lundstedt y E. Flygt, "Coloured cullet: from waste to resource", Glass International, 55 y 56 pp. (2008)

5. M. Aineto y A. Acosta, "Las escorias de la central térmica GICC ELCOGAS como materia prima para la síntesis de materiales vitrocerámicos. Parte 1: Comportamiento en fusión de las escorias GICC y obtención del vidrio original", Boletín de la Sociedad Española de Cerámica y Vidrio, No.6, Vol. 44, 399-404 pp. (2005)

6. M. Aineto, A. Acosta, J. Rincón y M. Romero, "Las escorias de la central térmica GICC ELCOGAS como materia prima para la síntesis de materiales vitrocerámicos. Parte 2: Síntesis y caracterización de los materiales vitrocerámicos", Boletín de la Sociedad Española de Cerámica y Vidrio, No. 5, Vol. 45, 28-32 pp. (2006)

7. W. Smith, "Fundamento de la ciencia e ingeniería de materiales", McGrawHill, 2da. Edición. (1993)

8. K. Budinski, "Engineering Materials, Properties and Selection", Prentice Hall, 5ta. Edición. (1996)

9. W. Zachariasen, "The atomic arrangement in glasses", Journal of the American Chemical Society, No 54, 3841-3851 pp. (1932)

10. J. Gibbs y E. DiMarzio, "Nature of the Glass Transition and the Glassy State, Journal of Chemical and Physics", Vol. 28, No. 3. (1958)

11. Z. Strnad, "Glass-ceramic materials, Encyclopedia Glass Science and Technology", No. 8, Editorial Elsevier, 268 pp. (1986)

12. A. Álvarez, "Estudio cinético de vitrocerámicos a partir de residuos industriales inorgánicos", Tesis Doctoral, Universidad Autónoma de Nuevo León. (2007)

13. S. Kalpakjian y S. Schmid, "Manufactura, ingeniería y tecnología”, Cuarta edición, Editorial Pearson Educación, 1152 pp. (2002)

14. M. Réamur, "The art of making a new type of porcelain by simple and easy methods of transforming glass into porcelain Part 1", Memories de I'Academie des Sciences, 139, 370-388 pp. (1739)

15. I. Kitalgorodsky y K. Bondarov, "Glass ceramics - A new universal material", Soviet Daily Newspaper, Pravda. (1963)

16. N. Seignes, A. Gouthier, D. Bulteel, M. Bautier, P. Reccurt y D. Damidat, "Effect of Pb-rich and Fe-rich entities during alteration of a partially vitrified metallurgical waste", Journal of Hazardous Materials, 149, 418-431 pp. (2007)

17. A. Karamarov y M. Pelino, "Cristallization phenomena in Fe-rich glasses", Journal Non-Crystal Solids, 281, 139-151 pp. (2001)

18. G. Calas, L. Cormier, L. Galaysis y V. Jollivet, "Structure-property relationships in multicomponent oxide glasses", Comptes Rendus Chimie, Número 12, Vol 5, 831-843 pp. (2002) 


\section{Introducción a los esmaltes cerámicos}

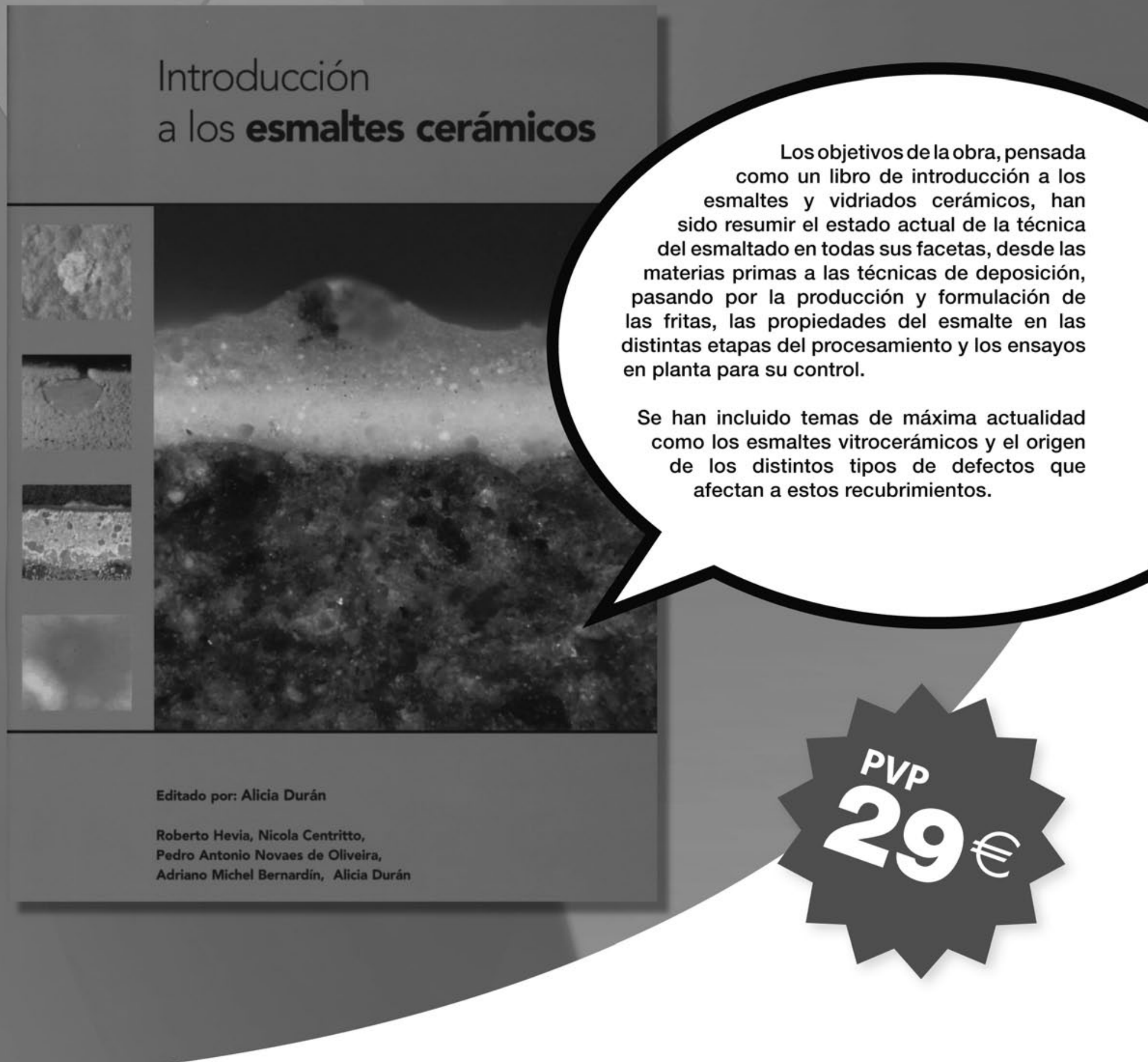

